\section{Ivabradine versus carvedilol in the management of palpitation with sinus tachycardia among recovered COVID-19 patients}

\author{
Kartik Pandurang Jadhav ${ }^{1 *}$ and Pankaj V Jariwala² \\ ${ }^{1}$ Interventional Cardiologist, Yashoda Hospital, Somajiguda, Hyderabad-500082, India \\ ${ }^{2}$ Senior Interventional Cardiologist, Department of Cardiology, Yashoda Hospital, Somajiguda, \\ Hyderabad-500082, India
}

\section{Abstract}

Introduction: One of the major complications among COVID-19 patients include cardiac arrhythmias. Commonest arrhythmia is sinus tachycardia which is usually associated with palpitation causing discomfort to patients. In this study, we present a comparative study of use of Ivabradine vs. Carvedilol for sinus tachycardia in post-COVID-19 infected patients.

Method: 50 consecutive recovered COVID-19 patients with sinus tachycardia were included in this open labelled RCT. 25 patients received Ivabradine and remaining 25 received Carvedilol. Single therapy non-responders were treated with Ivabradine with Atorvastatin.

Results: The mean age of all patients is $48.8 \pm 7.66$ years (Males $49.5 \pm 7.21$ years; Females $47.68 \pm 8.23$ years). The mean heart rate (MHR) of all patients is $125.52 \pm 9.07 / \mathrm{min}$ (Males $125.67 \pm 8.78 / \mathrm{min}$; Females $125.26 \pm 9.5 / \mathrm{min}$ ). After five days of single drug therapy the mean drop in the heart rate was $35.04 \pm 10.55 / \mathrm{min}$ (Males $34.41 \pm 9.71 / \mathrm{min}$; Females $36.05 \pm 11.72$ / $\mathrm{min}$ ), resulting in $27.88 \pm 8.11 \%$ (Males $27.38 \pm 7.56 \%$; Females $28.69 \pm 8.89 \%$ ) reduction in MHR. Among the two groups, the Carvedilol group showed improvement of MHR in 14(56\%) patients; whereas in Ivabradine group 18(72\%) patients improved out of 25 patients each ( $\mathrm{p}$ : $0.2385)$. In the Carvedilol group the MHR reduced from $128.6 \pm 8.44$ to $95.68 \pm 10.63(p<0.001)$, which is statistically significant; similarly, the Ivabradine group showed a MHR from $122.44 \pm 8.62$ to $85.28 \pm 10.52(p<0.001)$. The monotherapy therapy non-responders were treated with dualtherapy of (Ivabradine + Atorvastatin)

Discussion: Ivabradine is more effective in controlling heart rate compared to Carvedilol. Also, Ivabradine group scores very well in 'patient-satisfaction' with regards to symptom (palpitation) relief.

Conclusion: The COVID-19 sequelae of sinus tachycardia can be better controlled with Ivabradine when compared to Carvedilol.
More Information

*Address for Correspondence:

Dr. Kartik Pandurang Jadhav, Interventional Cardiologist, Yashoda Hospital, Somajiguda, Hyderabad- 500082, India, Tel: 8129013016 Email: drkartik303@gmail.com

Submitted: November 06, 2020 Approved: December 22, 2020 Published: December 23, 2020

How to cite this article: Jadhav KP, Jariwala PV. Ivabradine versus carvedilol in the management of palpitation with sinus tachycardia among recovered COVID-19 patients. J Cardiol Cardiovasc Med. 2020; 5: 176-180.

DOI: 10.29328/journal.jccm.1001107

Copyright: () 2020 Jadhav KP, et al. This is an open access article distributed under the Creative Commons Attribution License, which permits unrestricted use, distribution, and reproduction in any medium, provided the original work is properly cited.

Keywords: COVID-19; Recovered COVID-19; Sinus tachycardia; Carvedilol in COVID-19; Ivabradine in COVID-19

Check for updates

OPEN ACCESS

\section{Introduction}

On 31 $1^{\text {st }}$ December, 2019 a novel coronavirus was detected in Wuhan, a city in the Hubei Province of China; which was named Coronavirus disease-2019 (COVID-19). The causative agent behind this pandemic was designated as "Severe Acute Respiratory Syndrome - Coronavirus-2" (SARSCoV-2) [1]. From the day it was first detected, it has spread rapidly resulting in a global pandemic. This pandemic has presented an unprecedented challenge to public health, social structures, economic setup and food security. This has not only led to substantial loss of human life (1.68 million deaths till 20 ${ }^{\text {th }}$ December 2020), but has also led to long term sequelae among recovered COVID-19 patients thus putting tremendous strain on medical resources. Even the un-infected people have undergone incredible amount of psychological stress (depression, anxiety, fear psychosis, panic attacks), social stress (fear of social gatherings, ban on social events) and economical stress (loss of jobs, rise in poverty, drop in earnings, reduced governmental expenditure on developmental projects) [2-4]. 
The commonest presenting symptoms among the COVID-19 patients includes fever, fatigue, dry cough, anorexia, myalgia, dyspnoea and sputum production [5]. In an observational study in Germany, cardiovascular magnetic resonance imaging (cMRI) of recovered COVID-19 patients, revealed 78\% cardiac involvement and $60 \%$ had ongoing inflammation, irrespective of severity [6]. The arrhythmias are one of the common manifestations of this cardiac involvement. The arrhythmias are presumed to occur due to direct viral effect and partly due to systemic illness. In a cohort of 137 patients from Hubei province, $7.3 \%$ reported palpitation as presenting symptoms [7]. The exact prevalence of sinus tachycardia or for that matter various other arrhythmias among the recovered patients is not known [8]. Therefore, the treatment of the sinus tachycardia in recovered patients too is not formulated.

Our study tries to answer the above questions and determines whether a beta-blocker or a drug acting on sinus node is effective in controlling sinus tachycardia in recovered COVID-19 patients.

\section{Objectives of the study}

\section{Primary}

-To compare the effectiveness of Carvedilol v/s Ivabradine in the treatment for controlling heart rate for sinus tachycardia in recovered COVID-19 patients.

-To determine the effectiveness of Ivabradine with Atorvastatin combination in the patients with therapy nonresponders to either Carvedilol or Ivabradine single therapy.

\section{Secondary}

-To know the prevalence of sinus tachycardia and/or palpitations among the recovered COVID-19 patients.

\section{Methods}

\section{Study design}

This is an out-patient department (OPD) based, single centre, randomized, non-blinded and open labelled study. The executive committee, consisting of 2 experienced cardiologists oversaw the conduct of the study. The data obtained was tabulated using Microsoft Excel and statistical analysis was performed by an experienced bio-statistician at the medical centre. The manuscript was drafted by the executive committee members and looked for accuracy and completeness of reported data. The study was conducted on OPD basis with the written consent of patients.

\section{Study patients}

The inclusion criteria for the study included, patients with history recovery from COVID-19 infection (less than 2 weeks duration). The patients who complained of palpitations with or without sweating with heart rate of more than 100 beats/ minute were included in the study. All patients underwent
ECG, and those with sinus tachycardia were included. The diagnosis proven by reverse transcriptase polymerase chain reaction (RT-PCR) test and recovery too confirmed by RT-CPR test being negative. The high-resolution computed tomography (HR-CT) of chest showing CORADs score between 3 to 5. Patients with no previously known co-morbidities. Patients of both gender and age between 30 to 60 years.

The exclusion criteria included patients apart from the inclusion criteria. Patients who required invasive ventilation. Patients who had extensive lung fibrosis. Patients who had cardio-vascular complications like (myocardial infarction; intracardiac thrombosis; stress cardiomyopathy; vascular thrombosis; Stroke). Patients with renal complications (acute kidney injury; anuria) or severely deranged liver enzymes (SGOT/SGPT > 200 units/Litre). Patients with history of congenital heart diseases or ECG showing tachy-arrhythmias other than sinus tachycardia were excluded.

\section{Study treatment}

The first 50 consecutive patients satisfying above inclusion and exclusion criteria were randomized to either Ivabradine or Carvedilol drug therapy in a non-blinded fashion on OPD basis for the management of sinus tachycardia. The Carvedilol group received maximum tolerated dose of the drug (3.125 mg/6.25 mg/12.5 mg twice daily) for a period of 5 days; similarly, in Ivabradine group the dose received was $5 \mathrm{mg} /$ $10 \mathrm{mg}$ twice daily for a period of 5 days. All patients received multi-vitamins and zinc supplements. If the symptoms of palpitations/tachycardia are not reduced on single drug therapy within a period of 5 days, then they will be started on Ivabradine with Atorvastatin combination for a period of 5 days. The patients were advised to follow-up after 5 days in OPD.

\section{End points}

The success/effectiveness of the treatment therapy is defined as 'reduction of heart rate from the base level by $30 \%$ or more'. If the said target for heart rate is not achieved then therapy was changed and again looked for reduction in heart rate.

\section{Statistical analysis}

The JASP Team (2020). JASP (Version 0.14) [Computer software] was used to conduct statistical analysis. The findings were interpreted using descriptive statistics, including mean, median, ranges, standard deviation, chi square and percentages. The paired and unpaired t-test was used to compare the results between Ivabradine and Carvedilol group.

\section{Results}

\section{Study drugs and study patients}

Out of total 460 patients treated for COVID-19 at a tertiary care centre, $80(17.4 \%)$ patients presented to COVID-19 OPD 
with palpitation as symptom. Out of which 50 patients fulfilled the inclusion and exclusion criteria for the study. The 50 $(10.8 \%)$ patients were randomly divided into 2 groups of 25 each, who either received Carvedilol or Ivabradine as therapy for sinus tachycardia (Figure 1).

Out of 50 patients, 31 (62\%) are males and 19 (38\%) are females. None of them had any previously diagnosed co-morbidities. None of the patient had any complications associated with the drug dosages. All the patients got a 5-day therapy of single drug regimen.

The mean age of all patients is $48.8 \pm 7.66$ years (Males 49.5 \pm 7.21 years; Females $47.68 \pm 8.23$ years). The mean hear rate of all patients is $125.52 \pm 9.07 / \mathrm{min}$ (Males $125.67 \pm 8.78 / \mathrm{min}$; Females $125.26 \pm 9.5 / \mathrm{min}$ ). After five days of single drug therapy the mean drop in the heart rate was $35.04 \pm 10.55 / \mathrm{min}$ (Males $34.41 \pm 9.71 / \mathrm{min} ;$ Females $36.05 \pm 11.72 / \mathrm{min}$ ), resulting in $27.88 \pm 8.11 \%$ (Males $27.38 \pm 7.56 \%$; Females $28.69 \pm 8.89 \%$ ) reduction in heart rate.

The group of patients who had $30 \%$ or more reduction of the heart rate after starting single drug therapy within 5 days were considered as 'effective therapy'.

\section{Group comparison}

Among the two groups, the Carvedilol group showed improvement of tachycardia in 14 (56\%) patients; whereas in Ivabradine group 18(72\%) patients improved out of 25 patients each. No statistical significance noted (p: 0.2385). In the Carvedilol group the mean heart rate (MHR) reduced from $128.6 \pm 8.44$ to $95.68 \pm 10.63(p<0.001)$, which is statistically significant; similarly, the Ivabradine group showed a mean heart rate from $122.44 \pm 8.62$ to $85.28 \pm 10.52(p<0.001)$. The co-relation graph showing clustering of cases during pre- and post-therapy period. The density descriptive graph shows pre-therapy equal distribution of cases from 100 to $140 /$ min heart rate of cases, which later on after post-therapy clustering of cases below 100/min on heart rate. None of the patients had any side-effects related to the drug intake (Figure 2 \& Table 1).

The Odds ratio between the Ivabradine group and Carvedilol group, keeping carvedilol as control is 2.02 (p: 0.2416, CI: 0.6226-6.5569). The relative risk is 1.57 .

\section{Therapy non-responders}

Among the 50 patients in Carvedilol and Ivabradine group, 18 patients failed to respond to the single drug therapy, following which they were placed in Ivabradine and Atorvastatin group. Out of the 18 patients placed on dual therapy, $16(88.89 \%)$ patients had improved heart rate, whereas $2(11.11 \%)$ patients failed to respond to the therapy even after giving it for a period of 15 days. This improvement was statistically significant $(p<0.001)$ (Table 1$)$.

\section{Drug side-effects}

None of the participating subjects experienced any sideeffects of the drugs administered.

\section{Study design}

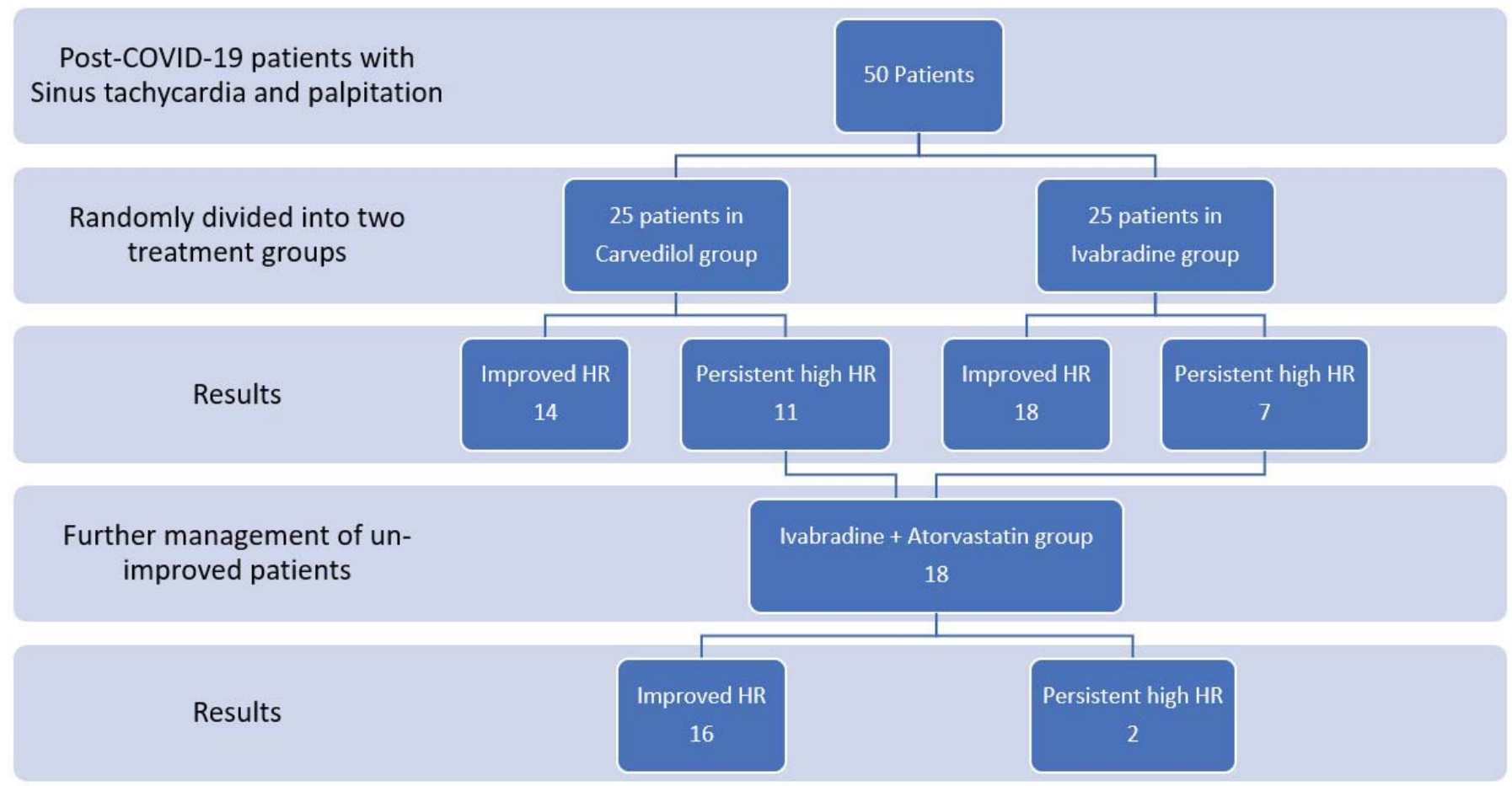


(A)

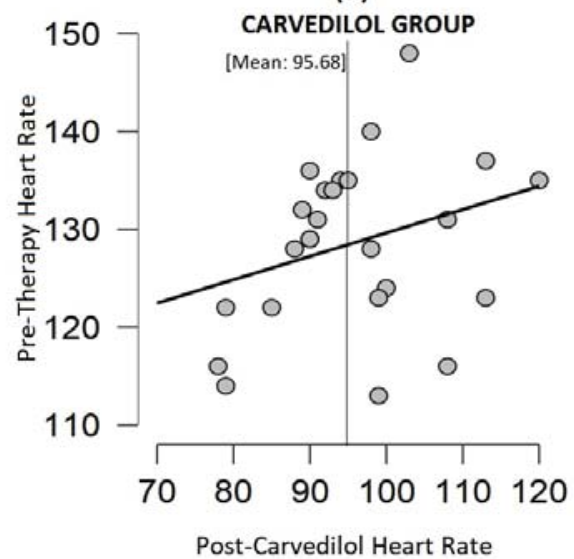

(B)

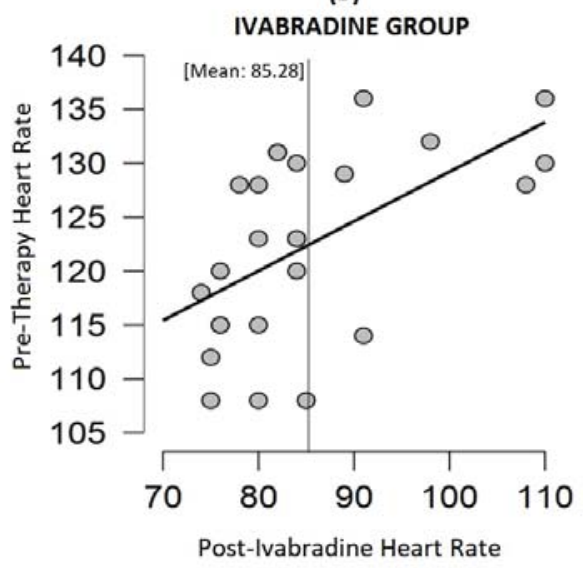

(C)

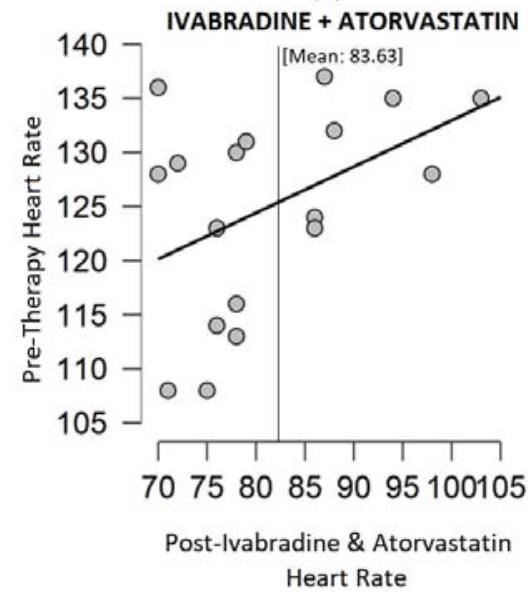

Figure 2: Descriptive plots showing Mean and Standard deviation of Heart rate in Pre- and Post-Therapy in Carvedilol group (A); Ivabradine group (B) and Ivabradine + Atrovastatin group (C).

Table 1: Carvedilol (A) and Ivabradine (B) Therapy Group table showing Mean with Standard Deviation of Age, Pre-therapy Heart Rate, Difference between Pre- and PostTherapy Heart Rate and Percentage change of Heart Rate in relation to therapeutic Effectiveness and Ineffectiveness after drug therapy. Paired Student T-Test (C) of Pre- and Post-therapy (Carvedilol, Ivabradine and Ivabradine with Atorvastatin) for Sinus Tachycardia.

\begin{tabular}{|c|c|c|c|c|c|}
\hline \multicolumn{6}{|c|}{ Carvedilol Therapy Group } \\
\hline Therapy Outcome & Age (Years) & \multicolumn{2}{|c|}{ Pre-therapy Heart-Rate (per minute) } & Heart-Rate reduction Post-Therapy & Percentage reduction Post-Therapy \\
\hline Effective & $46.64 \pm 7.92$ & \multicolumn{2}{|r|}{$130.07 \pm 8.89$} & $40.85 \pm 2.89$ & $31.44 \pm 1.51$ \\
\hline Ineffective & $46.72 \pm 7.91$ & \multicolumn{2}{|r|}{$126.72 \pm 7.42$} & $22.81 \pm 10.16$ & $17.79 \pm 7.49$ \\
\hline \multicolumn{6}{|c|}{ Ivabradine Therapy Group } \\
\hline Therapy Outcome & Age (Years) & \multicolumn{2}{|c|}{ Pre-therapy Heart-Rate (per minute) } & Heart-Rate reduction Post-Therapy & Percentage reduction Post-Therapy \\
\hline Effective & $52.11 \pm 6.58$ & \multicolumn{2}{|r|}{$122.5 \pm 7.48$} & $41.94 \pm 5.07$ & $34.16 \pm 2.81$ \\
\hline Ineffective & $48 \pm 6.23$ & \multicolumn{2}{|r|}{$122.28 \pm 11.02$} & $24.85 \pm 4.61$ & $20.46 \pm 3.95$ \\
\hline \multicolumn{6}{|c|}{ Paired Student T-Test } \\
\hline & & & & \multicolumn{2}{|c|}{$95 \% \mathrm{Cl}$ for Mean Difference } \\
\hline & $\mathbf{t}$ & $p$ & Mean Difference \pm SE & Lower & Upper \\
\hline Pre- \& Post Carvedilol & 14.128 & $<.001$ & $32.920 \pm 2.330$ & 28.111 & 37.729 \\
\hline Pre- \& Post-Ivabradine & 19.942 & $<.001$ & $37.160 \pm 1.863$ & 33.314 & 41.006 \\
\hline Pre- \& Post-Iva+Atorva & 18.101 & $<.001$ & $43.611 \pm 2.409$ & 38.528 & 48.694 \\
\hline
\end{tabular}

\section{Discussion}

COVID-19 infection can lead to increased risk of cardiac arrhythmias. There are many hypothesis and mechanism explaining this. The imbalance between metabolic demand and reduced cardiac reserve [9], direct myocardial damage and increased inflammatory response, increased the risk of cardiac arrhythmias [10]. The hypoxia caused due to direct viral damage of lung tissue, can lead to arrhythmias [11]. The tendencies to have arrhythmias (especially sinus tachycardia) even after recovery remains quite high.

This study shows that sinus tachycardia in recovered COVID-19 patients is quite common and management of the same should include use of Ivabradine, as it controlled tachycardia in significant number of patients as compared to Carvedilol. These significant benefits were achieved without experiencing any side-effects from the drug administration. The benefits were seen regardless of age and gender among the subjects.

The beta-blockers act on atrio-ventricular node (AVN), and thus reduces the heart rate. Our study included Carvedilol, which is the frequently prescribed beta-blocker in cardiology practice. Ivabradine drug acts on sinus node by inhibiting $I_{\mathrm{f}}$ sodium channels, thus achieving negative chronotropic effect but no inotropic manifestation on heart [12]. Both these medications are prescribed to control heart rate during tachycardia.Our study did a head-to-head comparative analysis of Carvedilol v/s Ivabradine in recovered COVID-19 patients with sinus tachycardia with palpitations. And the results show that Ivabradine fared better than Carvedilol in such patients. The cohort treated with Ivabradine monotherapy was twice as likely to get therapeutic improvement in MHR when compared to Carvedilol treated cohort.

Among the therapy non-responders, we chose to give Atorvastatin (HMG Co-A reductase inhibitor) of $20 \mathrm{mg}$ along with Ivabradine $10 \mathrm{mg}$ twice daily. Statins inhibit MYD88, thus stabilizing MYD88 levels during infection, in-turn prevents devastating inflammatory response during COVID-19 infection [13]. Also statins are known to have anti-arrhythmic effect and reduce heart rate variability [14]. These above mechanisms of 
statins can be protective in nature against COVID-19 [15]. And thus, it was included in the study to look for controlling sinus tachycardia among non-responders along with Ivabradine as double therapy. Among the 18 non-responders, 16 (88.9\%) patients improved proving the efficacy of the dual therapy.

\section{Conclusion}

We still lack comprehensive treatment guidelines for treatment of arrhythmias during COIVD-19 infection and also among recovered COVID-19 patients. The prevalence of sinus tachycardia with palpitation among the recovered COVID-19 patients is quite high at $17.4 \%$. Both Carvedilol and Ivabradine have to be effective in controlling the heart rate, but Ivabradine is superior and twice as likely to control heart rate when compared to Carvedilol. The mono-therapy, non-responders can be better treated with dual therapy (Ivabradine and Atorvastatin).

\section{Highlights}

- Majority of COVID-19 patients suffer cardiac sequela during and after 'recovery' phase.

- The Commonest sequela include palpitation with sinus tachycardia.

- The incidence of sinus tachycardia among the recovered COVID-19 patients is high (17.5\%).

- Ivabradine is superior (2 times likely to improve MHR) to Carvedilol in controlling the heart rate.

\section{References}

1. WHO Timeline - COVID-19. WHO official website. 2020. https://www. who.int/news-room/detail/27-04-2020-who-timeline---covid-19

2. Sharma S, Singh G. Prevalence and Global Impact of 2019-nCoV Zoonotic Viruses on China and World: Consequences of Chaotic and Stressed Environment. Int J Computer Applications. 2020; 12: 16.

3. Sharma S, Sharma M, Singh G. A chaotic and stressed environment for 2019-nCoV suspected, infected and other people in India: Fear of mass destruction and causality. Asian J Psychiatr. 2020; 51: 102049. PubMed: https://pubmed.ncbi.nlm.nih.gov/32278890/

4. Gautam R, Sharma M. 2019-nCoV pandemic: A disruptive and stressful atmosphere for Indian academic fraternity. Brain Behav Immun. 2020; 88: 948-949.

PubMed: https://pubmed.ncbi.nlm.nih.gov/32289366/
5. Wang D, Hu B, Hu C, Zhu F, Liu X, et al. Clinical Characteristics of 138 Hospitalized Patients With 2019 Novel Coronavirus-Infected Pneumonia in Wuhan, China. JAMA. 2020; 323: 1061-1069.

PubMed: https://pubmed.ncbi.nlm.nih.gov/32031570/

6. Puntmann VO, Carerj ML, Wieters I, Fahim M, Arendt C, et al. Outcomes of Cardiovascular Magnetic Resonance Imaging in Patients Recently Recovered From Coronavirus Disease 2019 (COVID-19). JAMA Cardiol. 2020; 5: 1265-1273.

PubMed: https://pubmed.ncbi.nlm.nih.gov/32730619/

7. Liu K, Fang YY, Deng Y, Liu W, Wang MF, et al. Clinical characteristics of novel coronavirus cases in tertiary hospitals in Hubei Province. Chin Med J. 2020; 133: 1025-1031.

PubMed: https://pubmed.ncbi.nlm.nih.gov/32044814/

8. Lakkireddy DR, Chung MK, Gopinathannair R, Patton KK, Gluckman TJ et al. Guidance for Cardiac Electrophysiology During the COVID-19 Pandemic from the Heart Rhythm Society COVID-19 Task Force; Electrophysiology Section of the American College of Cardiology; and the Electrocardiography and Arrhythmias Committee of the Council on Clinical Cardiology, American Heart Association. Heart Rhythm. 2020; 17: e233-e241.

PubMed: https://pubmed.ncbi.nlm.nih.gov/32247013/

9. Xiong TY, Redwood S, Prendergast B, Chen M. Coronaviruses and the cardiovascular system: acute and long-term implications. Eur Heart $\mathrm{J}$. 2020; 41: 1798-1800.

PubMed: https://pubmed.ncbi.nlm.nih.gov/32186331/

10. Babapoor-Farrokhran S, Gill D, Walker J, Rasekhi RT, Bozorgnia B, et al. Myocardial injury and COVID-19: Possible mechanisms. Life Sci. 2020; 253: 117723.

PubMed: https://pubmed.ncbi.nlm.nih.gov/32360126/

11. Kochi AN, Tagliari AP, Forleo GB, Fassini GM, Tondo C. Cardiac and arrhythmic complications in patients with COVID-19. J Cardiovasc Electrophysiol. 2020; 31: 1003-1008.

PubMed: https://pubmed.ncbi.nlm.nih.gov/32270559/

12. Tse S, Mazzola N. Ivabradine (Corlanor) for Heart Failure: The First Selective and Specific I f Inhibitor. PT. 2015; 40: 810-814.

PubMed: https://pubmed.ncbi.nlm.nih.gov/26681903/

13. Totura AL, Whitmore A, Agnihothram S, Schäfer A, Katz MG, et al. Toll-Like Receptor 3 Signaling via TRIF Contributes to a Protective Innate Immune Response to Severe Acute Respiratory Syndrome Coronavirus Infection. MBio. 2015; 6: e00638-15.

PubMed: https://pubmed.ncbi.nlm.nih.gov/26015500/

14. Chen WR, Liu HB, Sha Y, Shi Y, Wang H, et al. Effects of Statin on Arrhythmia and Heart Rate Variability in Healthy Persons With 48 Hour Sleep Deprivation. J Am Heart Assoc. 2016; 5: e003833. PubMed: https://pubmed.ncbi.nlm.nih.gov/27799236/

15. Kow CS, Hasan SS. Meta-analysis of Effect of Statins in Patients with COVID-19. Am J Cardiol. 2020; 134: 153-155.

PubMed: https://www.ncbi.nlm.nih.gov/pmc/articles/PMC7419280/ 